\title{
Comprehensive Analysis of the Relationship Between Metabolic Reprogramming and Immune Function in Prostate Cancer
}

\author{
Weijie Xie $\mathbb{D}^{\prime}$ \\ Huan Guo' \\ Jiawei Zhang' \\ $\mathrm{Li} \mathrm{Hu}{ }^{2}$ \\ Yuqi Wu (1) \\ Xiangwei Wang ${ }^{1,3}$ \\ 'Department of Urology \& Carson \\ International Cancer Center, Shenzhen \\ University General Hospital \& Shenzhen \\ University Clinical Medical Academy \\ Center, Shenzhen University, Shenzhen, \\ People's Republic of China; ${ }^{2}$ Department \\ of Physiology, Shantou University of \\ Medical College, Shantou, People's \\ Republic of China; ${ }^{3}$ Department of \\ Urology, 3rd Affiliated Hospital and \\ Department of Perioperative Medicine of \\ Southern University of Science and \\ Technology, Southern University of \\ Science and Technology, Shenzhen, \\ People's Republic of China
}

Purpose: Prostate cancer is the most common malignant urinary tumor among men. Treatments are currently unsatisfactory for advanced prostate cancer. Cancer biology remains the basis for developing new antitumor drugs. Therefore, it is crucial to study the metabolic reprogramming, immune microenvironment, and immune evasion of tumors. This study aimed to clarify the relationship between tumor glycolysis and immune function in prostate cancer.

Materials and Methods: We downloaded the gene expression matrix and clinical data of prostate cancer from The Cancer Genome Atlas. We studied the expression profiles and prognostic significance of glycolysis-related genes and used CIBERSORT to identify the proportion of tumor-infiltrating immune cells. Through differential gene expression analysis, gene ontology analysis, Kyoto Encyclopedia of Genes and Genomes analysis, gene set enrichment analysis, and correlation analysis, we further explored the relationship between glycolytic activity and immune function. We also performed immunohistochemistry, Western blot and RT-qPCR experiments using human prostate cancer tissue and cell lines to verify the expression of some glycolytic genes, macrophage infiltration and polarization.

Results: Among glycolysis-related genes, the expression of SLC16A3 in prostate cancer tissues was lower than that in normal tissues, but its high expression was associated with poor prognosis. In the high SLC16A3 expression group, several glycolysis-related genes also showed high expression, which was confirmed by immunohistochemistry experiments and Western blot. In high-glycolysis group, the expression of immune-related genes and the interleukin-17 (IL-17) signaling pathway were upregulated. $\mathrm{CD}^{+} \mathrm{T}$ cells, regulatory $\mathrm{T}$ cells, macrophages, and other immune cells were highly enriched. Among them, M2 macrophage infiltration was associated with poor prognosis.

Conclusion: The enhanced glycolytic activity of prostate cancer may contribute to the formation of a pro-tumor immune microenvironment. The IL-17 signaling pathway may play an important mediating role in the interaction between tumor glycolysis and immune function.

Keywords: glycolysis, tumor-infiltrating immune cells, IL-17 signaling pathway, TCGA

\section{Introduction}

Prostate cancer is a malignant tumor of the urinary system in men with high incidence. In 2018, there were about 1.3 million new cases of prostate cancer worldwide, resulting in 359,000 deaths and making it the fifth leading cause of cancer death. ${ }^{1}$ Prostate cancer is the most common malignant tumor and the second leading cause of death in the United States, accounting for 174,650 estimated new cases and 31,620 estimated deaths in $2019 .{ }^{2}$ In China, with the increasing use of 
ultrasound-guided prostate biopsy, the incidence of prostate cancer has increased sharply, ${ }^{3}$ with an estimated 72,000 new cases and 31,000 deaths in $2015 .{ }^{4}$ Compared with other malignant tumors, the progression of prostate cancer is relatively slow, resulting in a 5-year survival rate of $>80 \% .{ }^{5,6}$ However, although hormone deprivation therapy can inhibit progression of advanced prostate cancer, this effect cannot be sustained for a long time. Since then, the serum level of prostate-specific antibody rises rapidly again, and the tumor progresses to the stage of castrationresistant prostate cancer (CRPC), which eventually leads to death.

The metabolism reprogramming is an important sign of cancer. Most cancer cells exhibit an increase in glucose consumption and glycolysis rate and the formation of the final product lactate, even in aerobic conditions. This phenomenon is called "Warburg effect", ${ }^{7,8}$ which provides cancer cells with multiple survival advantages and regulates the metabolism and function of neighboring cells in the tumor microenvironment (TME). However, the phenomenon called the "reverse Warburg effect" also exists. Some cancer cells may exhibit oxidative phenotype, while stromal cells can become glycolytic. The two kinds of cells work together to boost tumor progression. ${ }^{9}$ Regardless of the metabolism mode, lactate is an important mediator of metabolic interaction between cancer cells and TME. ${ }^{10}$ Early prostate cancer tends to enhance oxidative phosphorylation and fat production but limit glycolysis. However, with tumor development, prostate cancer cells begin to exhibit the Warburg effect. As tumor aerobic glycolysis promotes malignant transformation and tumor progression, it has a potential significance for prognosis judgement and cancer treatment. ${ }^{11-14}$

As an important part of the TME, tumor-infiltrating immune cells (TIICs) can change the immune microenvironment of tumors. Studies have shown that TIICs can interact with tumor cells and influence carcinogenesis and tumor progression, which makes targeting TIICs a promising method of antitumor therapy. ${ }^{15,16}$ The interaction between prostate cancer and TIIC has been proved by experiments. Nardone et al found that in prostate cancer patients who underwent salvage radiotherapy after biochemical recurrence, the expression of FoxP3, CCR7, or PD-1 by tumorinfiltrating $\mathrm{T}$ lymphocytes was associated with progressionfree survival and overall survival. ${ }^{17}$ Erlandsson et al reported that a large number of M2 macrophages in the prostate cancer microenvironment increased the probability of prostate cancer mortality. ${ }^{18}$
The highly acidic microenvironment caused by tumor glycolysis may affect the infiltration of immune cells, ultimately severely damaging the function of the immune system itself and promoting the growth, dissemination, and immune escape of primary tumors. ${ }^{19,20}$ In in vitro experiments, lactate could damage the maturation and differentiation of dendritic cells (DCs) and induce a suppressor phenotype. ${ }^{21,22}$ In vitro experiments also proved that an acidic $\mathrm{pH}$ can inhibit $\mathrm{T}$ cell responses, including the elimination of IFN- $\gamma$ and TNF- $\alpha$ secretion. ${ }^{23}$ However, based on clinical data in prostate cancer research, there are few comprehensive analyses of the relationship between tumor glycolysis, TIICs, and clinical characteristics. This study, therefore, aimed to investigate the status of glycolysis and immunity in prostate cancer using transcriptome and clinical data from The Cancer Genome Atlas (TCGA) project, to identify the relationship between tumor glycolysis and TIICs and reveal the potential role of TIICs in the prognosis of prostate cancer.

\section{Materials and Methods Data Preparation for Preprocessing Transcriptome and Clinical Data}

Overall gene expression profiles and clinical data of patients were downloaded from the TCGA database (https://portal.gdc.cancer.gov/). We implemented a multiarray average algorithm and preprocessed the raw data. We then used the voom method to convert mRNA sequencing data to a microarray result. ${ }^{24}$ Furthermore, we used "limma" package of R to standardize the mRNA sequencing results. Subsequently, further analysis was performed with 15,514 gene expression datasets obtained from 52 normal tissue samples and 498 tumor tissues. Clinicopathological data, such as tumor stage, Gleason grade, survival status, and survival time, were obtained from the TCGA database.

\section{Differentially Expressed Genes and Enriched Pathways in Prostate Cancer}

The tumor samples were divided into low-glycolysis and high-glycolysis groups based on the median expression of SLC16A3. The differential gene expression analysis was obtained by using limma $\mathrm{R}$ package. Heatmap and volcano map were used to display differentially expressed genes (DEGs). The threshold of DEGs was $|\log 2 \mathrm{FC}| \geq 1$, with the adjusted $P$-value was $<0.05$. The exception was that when obtaining DEGs related to glycolysis between normal and 
tumor samples, the threshold of DEGs was $|\log 2 \mathrm{FC}| \geq 0.6$. DAVID (https://david.ncifcrf.gov/) was used for gene ontology (GO) analysis and Kyoto Encyclopedia of Genes and Genomes (KEGG) analysis. ${ }^{25}$ Gene set enrichment analysis (GSEA) based on WebGestalt (http://www. webgestalt.org/), ${ }^{26}$ with a false discovery rate (FDR) of $<0.05$ as the cutoff criterion, was performed to determine the specific signal pathway between the above two groups.

\section{Enumeration of TIICs}

CIBERSORT is an analytical tool that can use gene expression characteristics of 547 genes to estimate the abundance of cell types in mixed cell populations. ${ }^{27}$ It describes the characteristics of each immune cell subtype and uses a deconvolution algorithm to accurately quantify the different immune cell components. In this study, the LM22 signature file including 22 immune cell subtypes was used to illustrate the immune infiltration of each sample. After analyzing the differential gene expression profile on the CIBERSORT web (http://cibersort.stanford. edu), we obtained the distribution of 22 TIIC subtypes and the correlation coefficients, $P$-values, and root mean square errors (RMSEs).

\section{Patients and Specimens}

Human prostate cancer tissues were obtained from 60 patients of the Shenzhen University General Hospital between January 2018 and December 2020. All research procedures were approved by the Medical Ethical Committee of Shenzhen University Medical School according to the Declaration of Helsinki. The informed consents were obtained from all patients before tissue specimen collection.

\section{Cell Culture}

Human prostate cancer cell line PC3 and RAW246.7 macrophages were purchased from $\mathrm{BeNa}$ Culture Collection (Beijing, China). PC3 cells and RAW246.7 macrophages were cultured in PRMI 1640 medium (Invitrogen, Gaithersburg, MD, USA) containing 10\% fetal bovine serum (Invitrogen, USA) under the condition of $37^{\circ} \mathrm{C}$ and $5 \% \mathrm{CO}_{2}$. IL-4 $(10 \mathrm{ng} / \mathrm{mL})$ was used to stimulate RAW246.7 macrophages to obtain antiinflammatory macrophages (M2), ${ }^{28}$ while control macrophages (M0) were cultured in medium alone. Proinflammatory macrophages (M1) were induced by treating RAW246.7 macrophages with $50 \mathrm{ng} / \mathrm{mL}$ interferon (IFN)$\gamma$ and $10 \mathrm{ng} / \mathrm{mL}$ lipopolysaccharide (LPS). ${ }^{29}$

\section{PC3 Cell Transfection of siRNA Adenovirus}

PC3 cell lines were transfected with adenovirus vector packed with siRNA-MCT4 (RiboBio Corporation, Guangzhou, China), and polycoagulant amine (RiboBio Corporation, Guangzhou, China) was used as virus transfection efficiency enhancer. The specific sequence of interfering RNA fragment in siRNA-MCT4: 5'CCUACUCCGUCUACCUCUUTT-3' (sense) and 5'AAGAGGUAGACGGAGUAGGTT-3' (antisense); negative control (siRNA-NC) specific sequence: 5'UUCUCCGAACGUGUCACGUTT-3' (sense) and 5'ACGUGACACGUUCGGAGAATT-3' (antisense). The PC3 cell lines were laid on a 12-well plate and incubated overnight with $1 \mathrm{~mL}$ culture medium in each well. After 24 hours of incubation, the culture medium was removed and the mixture of $1 \mathrm{~mL}$ polycoagulant with culture medium was added to each well, and the concentration of polycondensed amine was $5 \mathrm{ug} / \mathrm{mL}$. Adenoviral particles were melted on the ice and the virus transfection began after complete melting. Virus particles with $1 \times 10^{7}$ viruses in each well were added to the culture medium to transfect cells. Then the 12-well plate was centrifuged at a low speed for 1.5 hours and incubated at $37^{\circ} \mathrm{C}$ for 24 hours. The culture medium was then removed and $1 \mathrm{~mL}$ culture medium without polyamine was added to each well, and the cells were incubated overnight. After 4 days of culture, the cells were collected and cryopreserved.

\section{PC3 and RAW264.7 Cocultured}

Tumor-associated macrophages (TAMs) were obtained by incubating RAW246.7 macrophages with PC3 cells or siMCT4-PC3 cells (PC3-TAM or si-MCT4-PC3-TAM). ${ }^{30}$ We developed a co-culture model by seeding RAW246.7 macrophages $\left(4 \times 10^{4} /\right.$ well $)$ in the lower chambers of the transwell plate with PC3 cells $\left(1 \times 10^{4} /\right.$ well $)$ in the upper chambers. After co-culturing for 84 hours, RAW246.7 macrophages were collected and mRNA analysis was performed by real-time quantitative PCR.

\section{Immunohistochemistry}

Human prostate cancer tissues were cut into $3-\mathrm{mm}$ sections. The paraffin sections were baked at $56^{\circ} \mathrm{C}$ for $2 \mathrm{~h}$ for dewaxing, boiled in citrate buffer to recover the antigen, and blocked with $3 \%$ hydrogen peroxide. Antibodies against monocarboxylate transporter 4 (MCT4) (dilution 
1:200; ProteinTech Group, Chicago, IL, USA), PFKFB3 (dilution 1:100; Abcam, UK), ENO2 (dilution 1:300; Servicebio, Wuhan, China), and M2 macrophage marker CD163 (dilution 1:100; Servicebio, Wuhan, China) were used to detect protein expression at $4{ }^{\circ} \mathrm{C}$ overnight, which was followed by incubation with HRP-conjugated secondary antibodies for $1 \mathrm{~h}$ at $37^{\circ} \mathrm{C}$. The $3^{\prime}$ diaminobenzidine kit (DAB; Dako Corporation, Carpinteria, CA, USA) was used for immune complex visualization. ImageJ software was used to measure the immunohistochemical staining intensity and the proportion of positive cells. Immunohistochemistry scores were independently evaluated by two doctors based on the method described in the article in a blinded manner. ${ }^{31}$ Scores of $1+$ and $2+$ were considered as low expression, while scores of $3+, 4+$, and $5+$ were regarded as high expression. ${ }^{25}$ The number of M2 macrophages was calculated for the entire tissue specimen under low magnification $(\times 10)$.

\section{Western Blotting}

The cells were divided into siRNA interference group (siMCT4 group), negative control group (si-NC group) and blank control group (NC group). The cells were harvested and lysed in RIPA Lysis and Extraction Buffer (Invitrogen, USA). The protein concentration of the lysate was determined by Pierce BCA protein assay kit (Thermo, Waltham, MA, USA). The lysate was electrophoretic on $8 \%$ SDS polyacrylamide gel and transferred to PVDF membrane (Millipore, Boston, MA, USA). The membrane was probed with the primary antibodies overnight at $4{ }^{\circ} \mathrm{C}$. The primary antibodies included MCT4 (dilution 1:1000; ProteinTech Group, Chicago, IL, USA), PFKFB3 (dilution 1:1000; Abcam, UK), ENO2 (dilution 1:1000; Servicebio, Wuhan, China) and $\beta$-actin (dilution 1:1000; CST, Boston, MA, USA). Then the membrane was incubated with the horseradish peroxidase-conjugated secondary antibodies (Zhongshan Jinqiao, Beijing, China) and scanned by Odyssey imaging system. The relative quantitative analysis was completed by lmageJ software, and the experiment was repeated three times.

\section{Real-Time Quantitative PCR (RT-qPCR)}

RNA was extracted from PC3 cells or macrophages by using Trizol reagent (Invitrogen, USA), and cDNA was generated by using RT-PCR kit (TaKaRa, Kyoto, Japan). Real-time quantitative PCR was performed by using the SYBR Green QuantiTect RT-PCR kit (Roche, South San
Francisco, CA), and each of the samples was analyzed three times. The sequences of the primers used for RTqPCR were shown as follows:

MCT4, 5'-TTTTGCTGCTGGGCAACTTCTTCTG-3' (sense) and 5'-TCACGTTGTCTCGAAGCATGGGTTT-3' (antisense);

CD206, 5'-GGCGAGCATCAAGAGTAAAGA-3' (sense) and 5'-CATAGGTCAGTCCCAACCAAA-3' (antisense);

Arg1, 5'-TTTTTCCAGCAGACCAGCTT-3' (sense) and 5'-CATGAGCTCCAAGCCAAAGT-3' (antisense);

iNOS, 5'-GAAACGCTTCACTTCCAATG-3' (sense) and 5'-AATCCACAACTCGCTCCAA-3' (antisense);

IL-6, 5'-AACGATGATGCACTTGCAGA-3' (sense) and 5'-TGGTACTCCAGAAGACCAGAGG-3' (antisense);

$\beta$-actin, 5'-CTAAGGCCAACCGTGAAAAG-3' (sense) and 5'-GGTACGACCAGAGGCATACA-3' (antisense).

\section{Statistical Analysis}

All analyses were performed using $\mathrm{R}$ version 3.6.3, and all statistical tests were two-sided. Differences in continuous data between the groups were compared using analysis of variance. Categorical data were analyzed using the chisquare test or Fisher's exact test. The prognostic significance of genes was analyzed by using Cox proportional hazards regression model. Spearman correlation analysis was used to assess the potential correlations. The KaplanMeier method was used to estimate the disease-free survival rate, and the Log rank test to evaluate the differences between the groups. $P<0.05$ was considered statistically significant.

\section{Results}

\section{Expression and Prognostic Significance of Glycolysis-Related Genes}

We selected 45 glycolysis-related genes and compared the expression profiles of these genes between normal tissues and tumor tissues. Results were showed in the heatmap and volcano map (Figure $1 \mathrm{~A}$ and B). Compared with expression in normal prostate tissue, the expression of SLC2A9, ENO2, SLC2A4, SLC16A7, ALDOC, PFKFB3, LDHB, and SLC16A3 in tumor tissue decreased, while that of HK2, SLC16A8, and FBP1 increased.

To find out the candidate glycolysis-related enzymes that may play an important role in the progression of prostate cancer, we investigated the relationship between clinical manifestations of prostate cancer and the 
expression of glycolysis-related genes. Univariate and multivariate Cox proportional hazards regression analyses were used to evaluate the gene-associated hazard ratio of disease-free survival. The results showed that SLC2A4 and SLC16A3 were independent risk factors for prostate cancer progression (Figure $1 \mathrm{C}$ and D). According to the SLC2A4 or SLC16A3 expression, the 550 prostate cancer patients from TCGA were divided into two groups. Survival analysis showed that the disease-free survival time was significantly shortened in the group with high SLC16A3 expression, which clarified the prognostic value of SLC16A3 (Figure 1E). In contrast, the group with high SLC2A4 expression predicted better disease-free survival (Figure 1F). We also found that a high SLC16A3 expression was positively correlated with a higher Gleason grade and worse diseasefree survival (Table 1).

\section{SLCI6A3 Expression and Glycolytic Activity}

SLC16A3 belongs to the solute carrier 16 family of genes and encodes MCT4. MCT4 shows high affinity for lactate, but low affinity for pyruvate, which leads to the conversion of pyruvate to lactate before export, promotes the outflow of lactate from glycolysis cells, and maintains high glycolysis flux in tumor cells. ${ }^{32}$ In view of the important role of SLC16A3 in glycolysis, we chose SLC16A3 for further studies. In order to further clarify whether the SLC16A3 expression can represent the level of glycolysis, we compared the expression of other glycolysis-related genes between the low SLC16A3 and high SLC16A3 expression group. The results demonstrated that PFKFB3, ALDOA, ALDOC, ENO2, ENO3, LDHA, LDHB, PCK2, PFKL, PFKP and PGM1 were significantly upregulated in the high

A
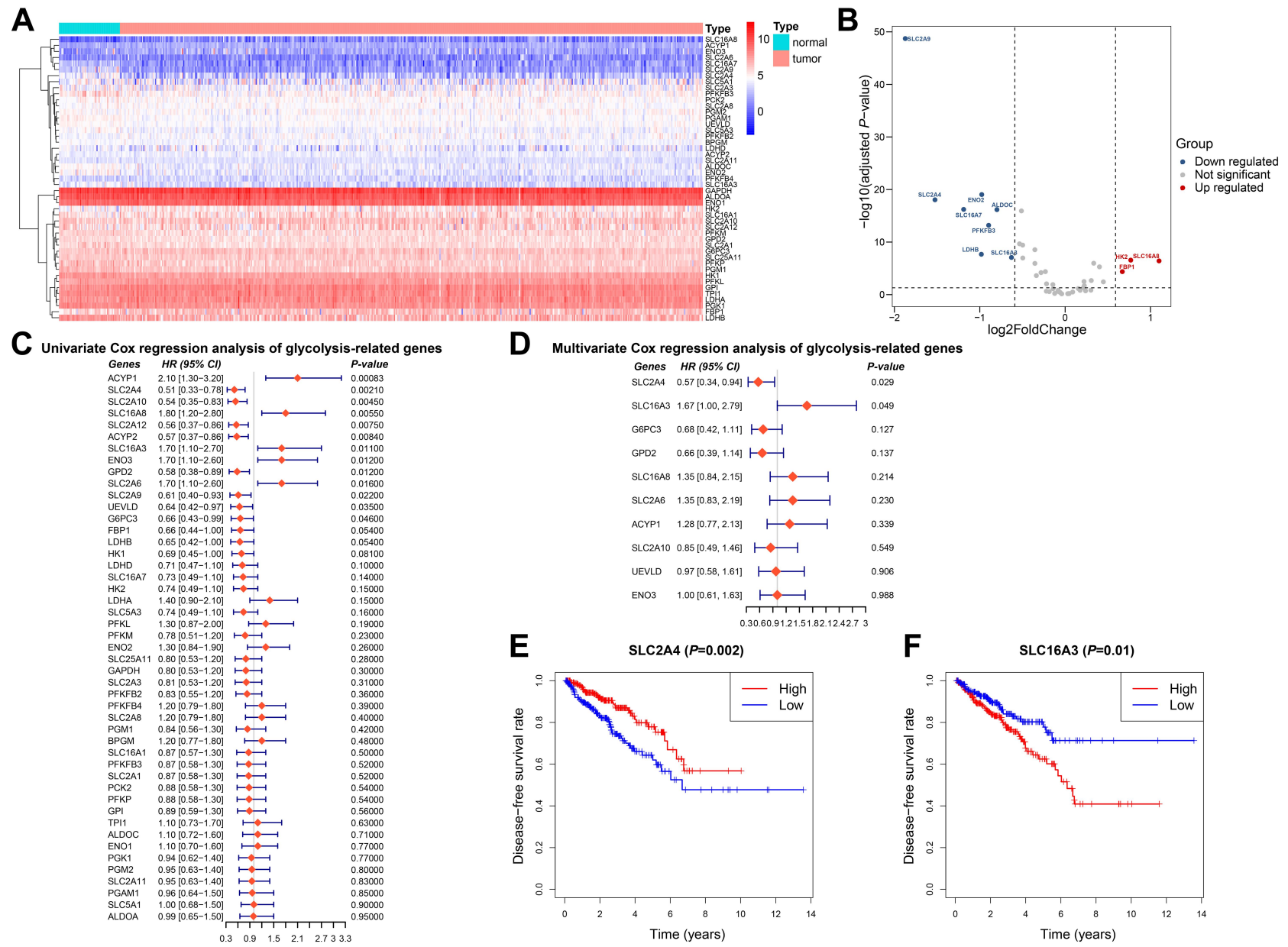

Figure I Expression and prognostic significance of glycolysis-related genes in prostate cancer. (A) Heatmap analysis of expression levels of 45 glycolysis-related genes. (B) Volcano plot for differentially expressed glycolysis-related genes. (C and D) Univariate and multivariate Cox regression of glycolysis-related genes. (E) Kaplan-Meier analysis of disease-free survival comparing low and high SLC2A4 expression. (F) Kaplan-Meier analysis of disease-free survival comparing low and high SLCI6A3 expression. 
Table I Clinical Characteristics of 489 Samples Base on SLCI6A3 Expression

\begin{tabular}{|c|c|c|c|}
\hline \multirow[t]{2}{*}{ Characteristics } & \multicolumn{3}{|c|}{ SLCI $6 A 3$} \\
\hline & $\begin{array}{l}\text { Low } \\
\text { Expression }\end{array}$ & $\begin{array}{l}\text { High } \\
\text { Expression }\end{array}$ & $P$-value \\
\hline Age & $60.30 \pm 7.01$ & $61.66 \pm 6.60$ & 0.028 \\
\hline T stage & & & 0.320 \\
\hline $\mathrm{T} 2$ & 102 & 83 & \\
\hline T3 & 136 & 152 & \\
\hline $\mathrm{T} 4$ & 5 & 5 & \\
\hline Tx & 2 & 4 & \\
\hline $\mathrm{N}$ stage & & & 0.109 \\
\hline No & 173 & 167 & \\
\hline $\mathrm{NI}$ & 31 & 46 & \\
\hline $\mathrm{Nx}$ & 41 & 31 & \\
\hline M stage & & & 0.343 \\
\hline Mo & 223 & 225 & \\
\hline MI & 0 & 2 & \\
\hline Mx & 22 & 17 & \\
\hline $\begin{array}{l}\text { Gleason grade } \\
\text { group }\end{array}$ & & & 0.005 \\
\hline 1 & 28 & 17 & \\
\hline 2 & 84 & 60 & \\
\hline 3 & 48 & 52 & \\
\hline 4 & 33 & 30 & \\
\hline 5 & 52 & 85 & \\
\hline Overall survival & & & 1 \\
\hline Alive & 243 & 242 & \\
\hline Dead & 2 & 2 & \\
\hline $\begin{array}{l}\text { Disease-free } \\
\text { survival }\end{array}$ & & & 0.003 \\
\hline Disease-free & 212 & 186 & \\
\hline $\begin{array}{l}\text { Recurred/ } \\
\text { progressed }\end{array}$ & 33 & 58 & \\
\hline
\end{tabular}

SLC16A3 expression group (Figure 2). All these genes are key genes in the glycolysis pathway. All above results suggested that SLC16A3 expression can reflect glycolytic activity, so the prostate cancer patients from TCGA can be divided into the low-glycolysis and high-glycolysis group according to the median expression of SLC16A3, respectively.

\section{Correlation Between Glycolytic Activity and Immune Function}

To study the biological differences between the low- and high-glycolysis groups, we analyzed the profiles of 260
DEGs between the two groups, including 239 upregulated genes and 21 downregulated genes (Figure 3A). Many cytokines and inflammatory mediators in the highglycolysis group were dysregulated and participated in the regulation of immune cell function (Figure 3B).

The enrichment analyses of GO and KEGG were carried out to reveal the gene functions and biological pathways. The functions related to immunity included GO:0006958 complement activation, classical pathway, GO:0006955 immune response, GO:0006956 complement activation, GO:0050871 positive regulation of B cell activation, GO:0006911 phagocytosis, engulfment, GO:0038096 Fc-gamma receptor signaling pathway involved in phagocytosis, GO:0006910 phagocytosis, recognition, GO:0050776 regulation of immune response, GO:0050853 B cell receptor signaling pathway, GO:0045087 innate immune response, GO:0006898 receptor-mediated endocytosis, GO:0006954 inflammatory response, GO:0006935 chemotaxis, GO:0042742 defense response to bacterium, GO:0038095 Fc-epsilon receptor signaling pathway, etc. (Figure 3C). The enriched signaling pathways were the hsa04621 NOD-like receptor signaling pathway, hsa04060 cytokine-cytokine receptor interaction, and hsa04668 TNF signaling pathway (Figure 3D).

\section{Upregulation of Immune-Related Pathways}

In view of the above findings, a GSEA study was performed to investigate the change of immune-related pathways in the high-glycolysis group. FDR of $<0.05$ was considered statistically significant. We found that two immune-related pathways were significantly upregulated, including the hsa04060 cytokine-cytokine receptor interaction and hsa04657 interleukin-17 signaling pathway (Figure 4A-C). Taken together, these findings indicated that high glycolytic activity was related to enhanced immune activity in prostate cancer.

\section{Relationship Between Glycolytic Activity and Immune Cell Infiltration}

To verify the relationship between glycolytic activity and immune function, we compared the degree of enrichment of immune cell types in the low- and high-glycolysis groups. In the high-glycolysis group, the degree of enrichment of $\mathrm{CD}^{+} \mathrm{T}$ cells, Tregs, monocytes, M1 macrophages, M2 macrophages, resting DCs, and activated 

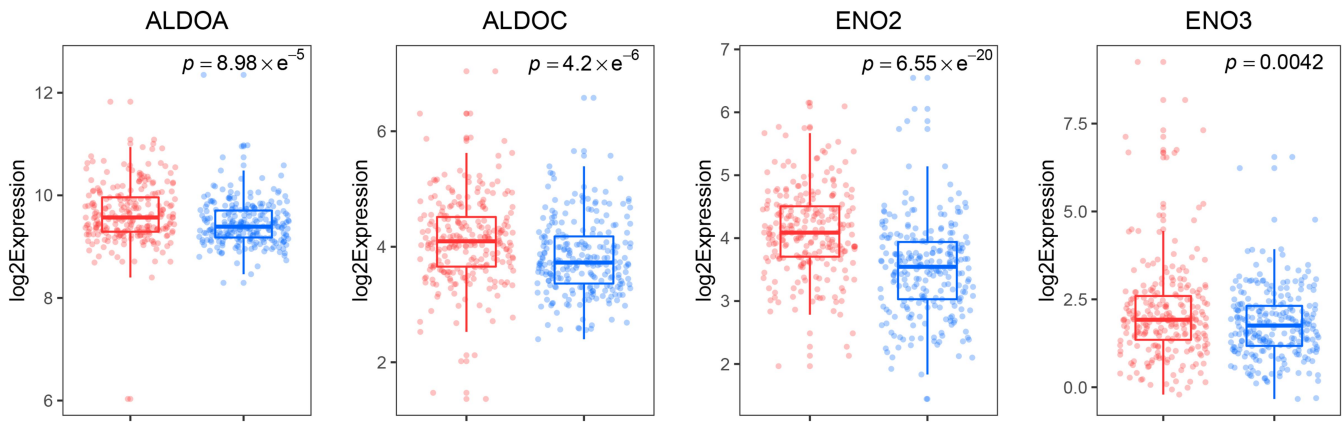

Group

审 High_SLC16A3_Expression

审 Low_SLC16A3_Expression
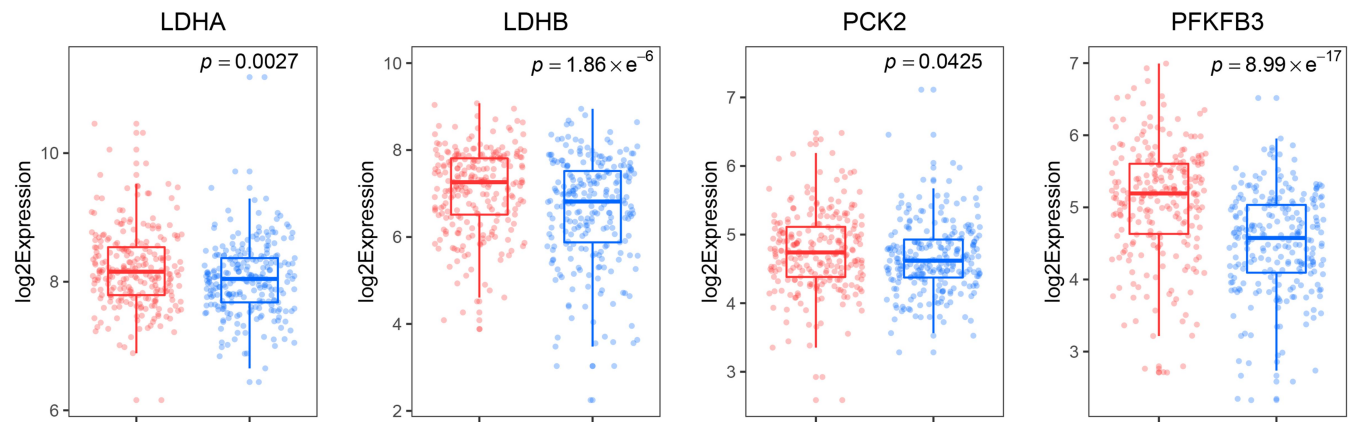

Group

由igh_SLC16A3_Expression

审 Low_SLC16A3_Expression
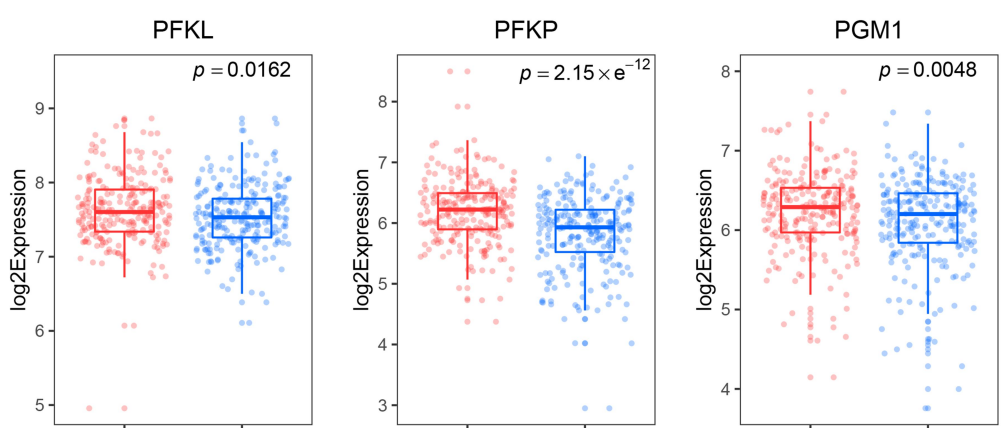

Group

宇 High_SLC16A3_Expression

审 Low_SLC16A3_Expression

Figure 2 The expression of SLCI6A3 can represent the glycolytic activity of prostate cancer to some extent. Box plots showed the expression of glycolysis-related genes ALDOA, ALDOC, ENO2, ENO3, LDHA, LDHB, PCK2, PFKFB3, PFKL, PFKP, and PGMI in the low and high SLCI6A3 expression groups.

mast cells was significantly higher. In contrast, the degree of enrichment of naïve B cells, plasma cells, and eosinophils in the high-glycolysis group was lower than that in the low-glycolysis group (Figure 5A). Additionally, SLC16A3 expression was positively correlated with the proportion of resting DCs $(r=0.147, P=0.001)$, M1 macrophages $(r=0.164, P<0.001)$, M2 macrophages $(r=0.132$, $P=0.003)$, activated mast cells $(r=0.116, P=0.009)$, monocytes $(r=0.211, \quad P<0.001), \mathrm{CD}^{+} \mathrm{T}$ cells $\quad(r=0.243$, $P<0.001)$, and Tregs $(r=0.284, P<0.001)$, but negatively correlated with that of naïve B cells ( $r=-0.205, P<0.001)$, plasma cells $(\mathrm{r}=-0.15, P<0.001)$, and eosinophils $(\mathrm{r}=$ $-0.146, P=0.001$ ) (Figure 5B). To distinguish tumorpromoting from antitumor immune cells, survival analysis was carried out to investigate the relationship between the survival time and the enrichment fraction of immune cells. The results showed that M2 macrophages were highly enriched in the high-glycolysis group, which predicted poor disease-free survival in prostate cancer (Figure 5C).

\section{Immunohistochemistry Results}

Considering that the high SLC16A3 expression was positively correlated with a higher Gleason grade, we evaluated the protein expression of MCT4 in human prostate cancer tissues. MCT4 expression was higher in tissues with a Gleason grade of 4-5 than in other tissues (Figure 6A). According to the expression score of MCT4, we defined the high- and low-expression groups in prostate cancer tissues as the high-glycolysis group and lowglycolysis group, respectively. Meanwhile, ENO2 protein 

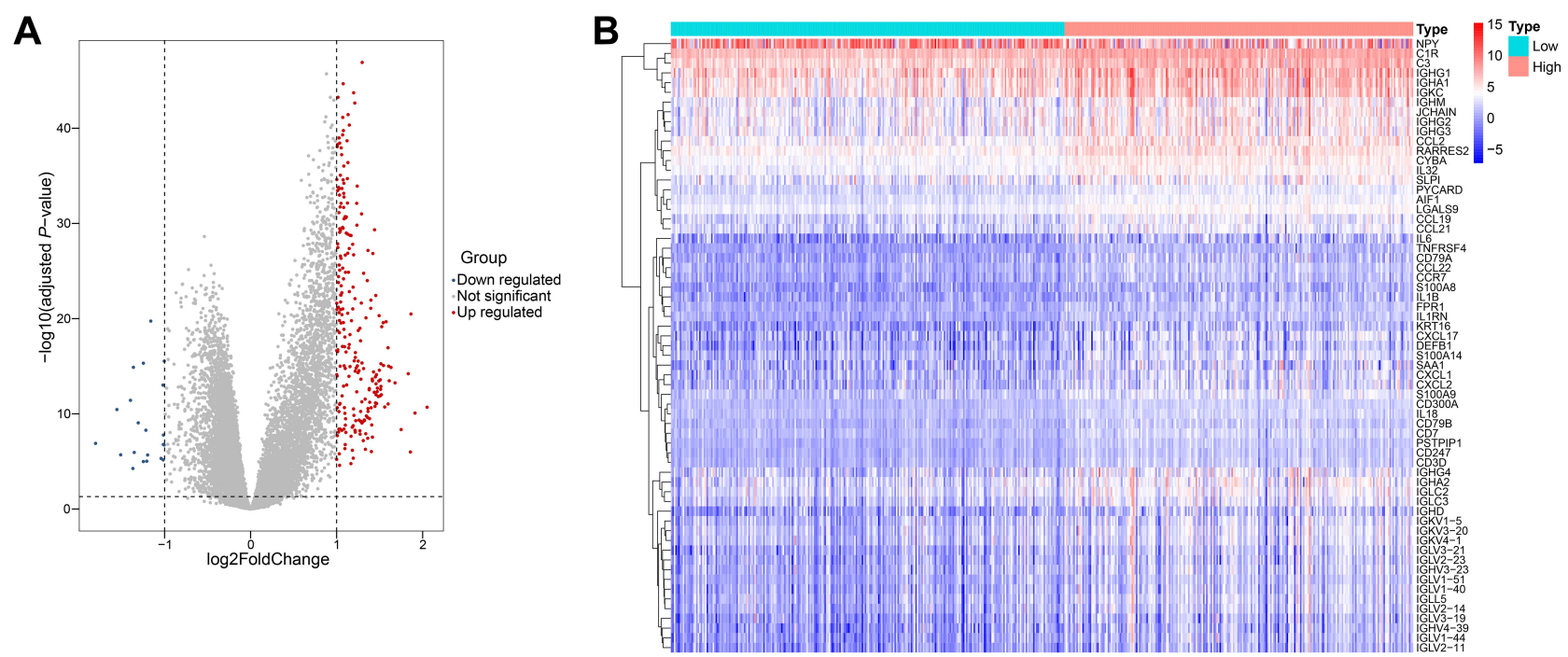

C

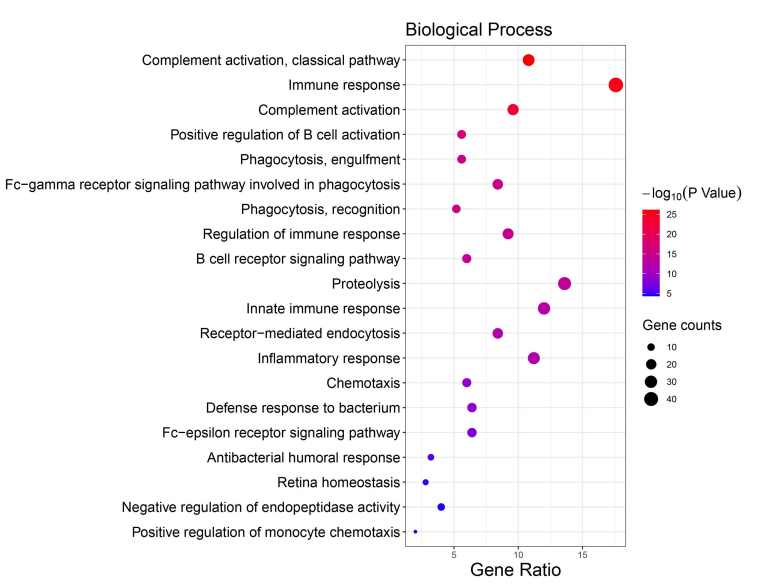

D

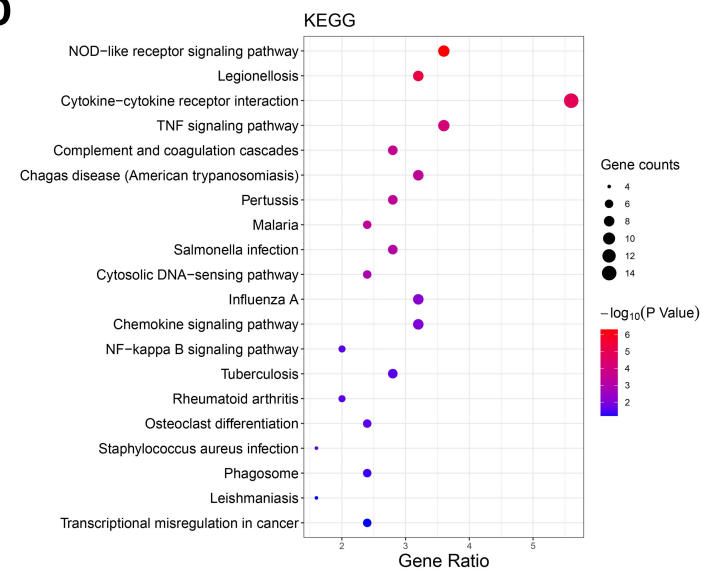

Figure 3 Correlation between glycolytic activity and immune function in prostate cancer. (A) Volcano plot for DEGs between the low- and high-glycolysis groups. (B) Heatmap analysis of dysregulated inflammation-related genes between the low- and high-glycolysis groups. (C and D) The top 20 most significant enrich Gene ontology (GO) and Kyoto Encyclopedia of Genes and Genomes (KEGG) terms with 260 DEGs analyses.

expression was higher in the high-glycolysis group than in the low-glycolysis group (Figure 6B). However, there was no difference in PFKFB3 expression between both groups (Figure 6C). Immunohistochemical analysis of CD163 showed that M2 macrophages were mainly located in the stroma near the tumor cells. The number of M2 macrophages in prostate cancer was significantly higher in the high-glycolysis group than in the low-glycolysis group (Figure 6D).

\section{Western Blot and RT-qPCR Results}

Furthermore, we conducted verification experiments on PC3 cell lines. We successfully constructed the si-NC and si-MCT4 PC3 cells, which were confirmed by RTqPCR, Western blot and fluorescence transfection experiments (Figure 7A, Supplementary Figure S1). The results (Figure 7A) showed that the gene and protein expression level of MCT4 in PC3 cells in si-MCT4 group was both significantly lower than that in $\mathrm{NC}$ group and si-NC group (all $P<0.001$ ). The results suggested that the expression of MCT4 in PC3 cells decreased significantly after si-MCT4 transfection, and the PC3 cells silencing MCT4 were successfully established. The results of Western blot also showed that protein expression levels of ENO2 and PFKFB3 in PC3 cells in si-MCT4 group were significantly lower than those in si-NC group and NC group $(P<0.001$ and $P<0.01$, respectively). The results suggested that the inhibition of MCT4 could reduce the glycolysis level of PC3 cells to some extent.

In order to determine whether PC3 cells and si-MCT4PC3 cells could change the phenotype of co-cultured macrophages, we constructed M1 and M2 macrophages for further experiments. As seen in Figure 7B, the gene expressions of anti-inflammatory markers Arg1 and 
A

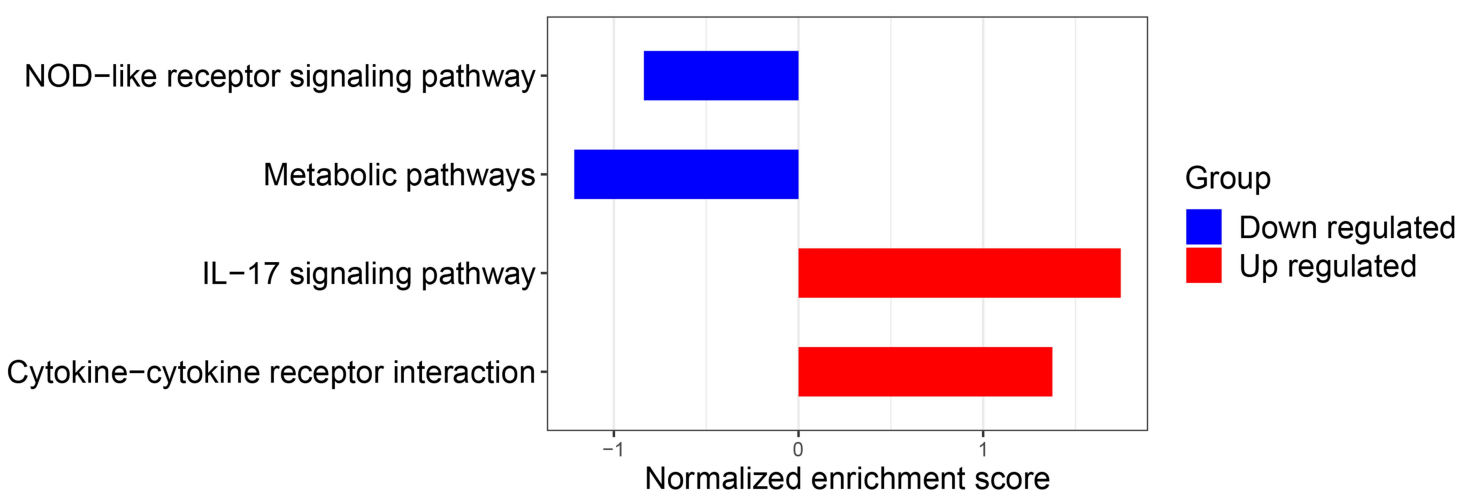

B

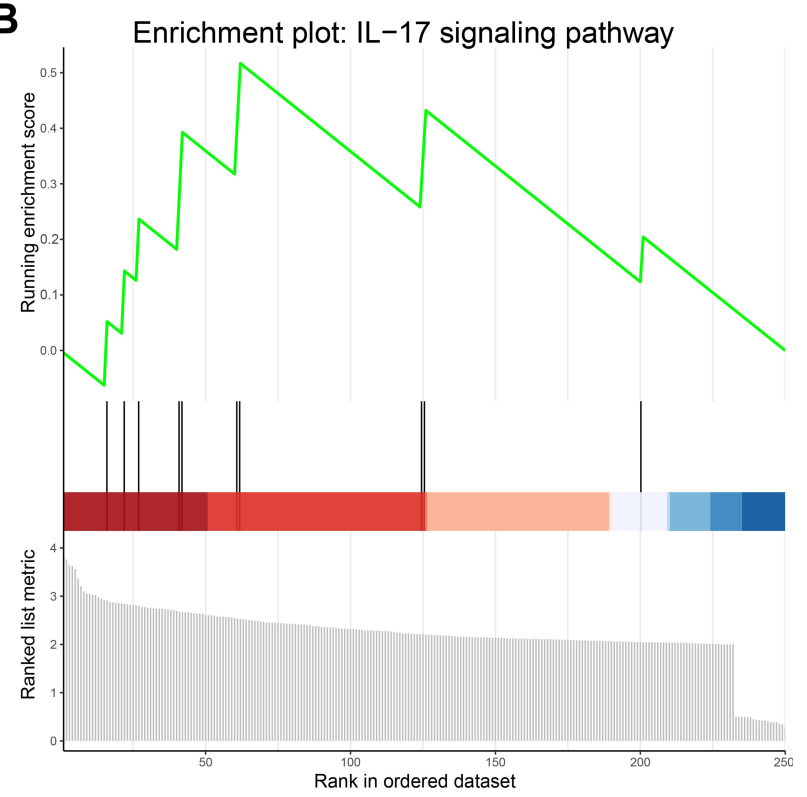

C Enrichment plot: Cytokine-cytokine receptor interaction

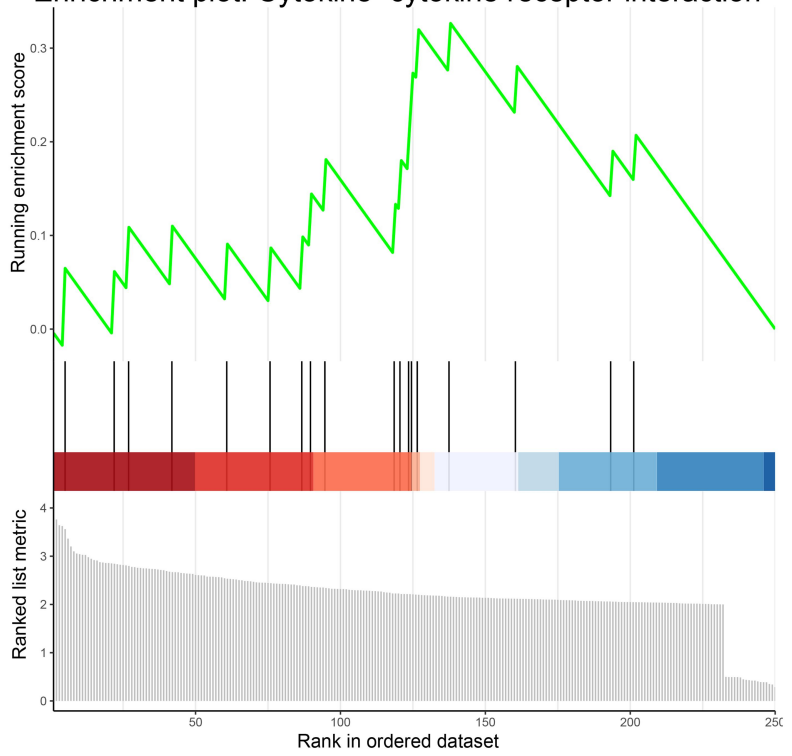

Figure 4 Upregulation of immune-related pathways in the high-glycolysis group. (A) The downregulated and upregulated pathways were obtained through gene set enrichment analysis (GSEA). (B and C) The GSEA charts showed the interleukin-17 (IL-I7) signaling pathway and cytokine-cytokine receptor interaction were significantly upregulated.

CD206 in macrophages induced by IL-4 were significantly increased (both $P<0.001$ ), while the gene expressions of pro-inflammatory markers IL-6 and iNOS were significantly increased in macrophages induced by IFN- $\gamma / \mathrm{LPS}$ compared with M0 macrophages $(P<0.001$ and $P<0.01$, respectively). Moreover, we cultured PC3 cells and RAW246.7 macrophages together in a transwell plate (Supplementary Figure S2). After macrophages were cocultured with PC3 cells, the gene expressions of Arg1 and CD206 in macrophages increased significantly compared with M0 macrophages (both $P<0.001$ ), but not IL-6 or iNOS, which indicated that PC3 cells promoted M2 polarization of macrophages. Moreover, when we silenced the expression of MCT4 in PC3 cells, the gene expressions of CD206 and Arg1 in si-MCT4-PC3-TAM were significantly downregulated compared with PC3-TAM (both $P<0.001$ ). These results suggested that the expression of MCT4 in PC3 cells played an important role in inducing M2 phenotype of macrophages.

\section{Discussion}

Tumorigenesis depends on the reprogramming of cell metabolism, which is characterized by a markedly increased consumption of glucose to generate energy by glycolysis, even under conditions of sufficient oxygen. The metabolic state of tumor cells also affects other cells in the TME, such as tumor-related fibroblasts and immune cells. Metabolites derived from tumor cells can affect the activation and function of immune cells, which ultimately promote the survival, proliferation and 

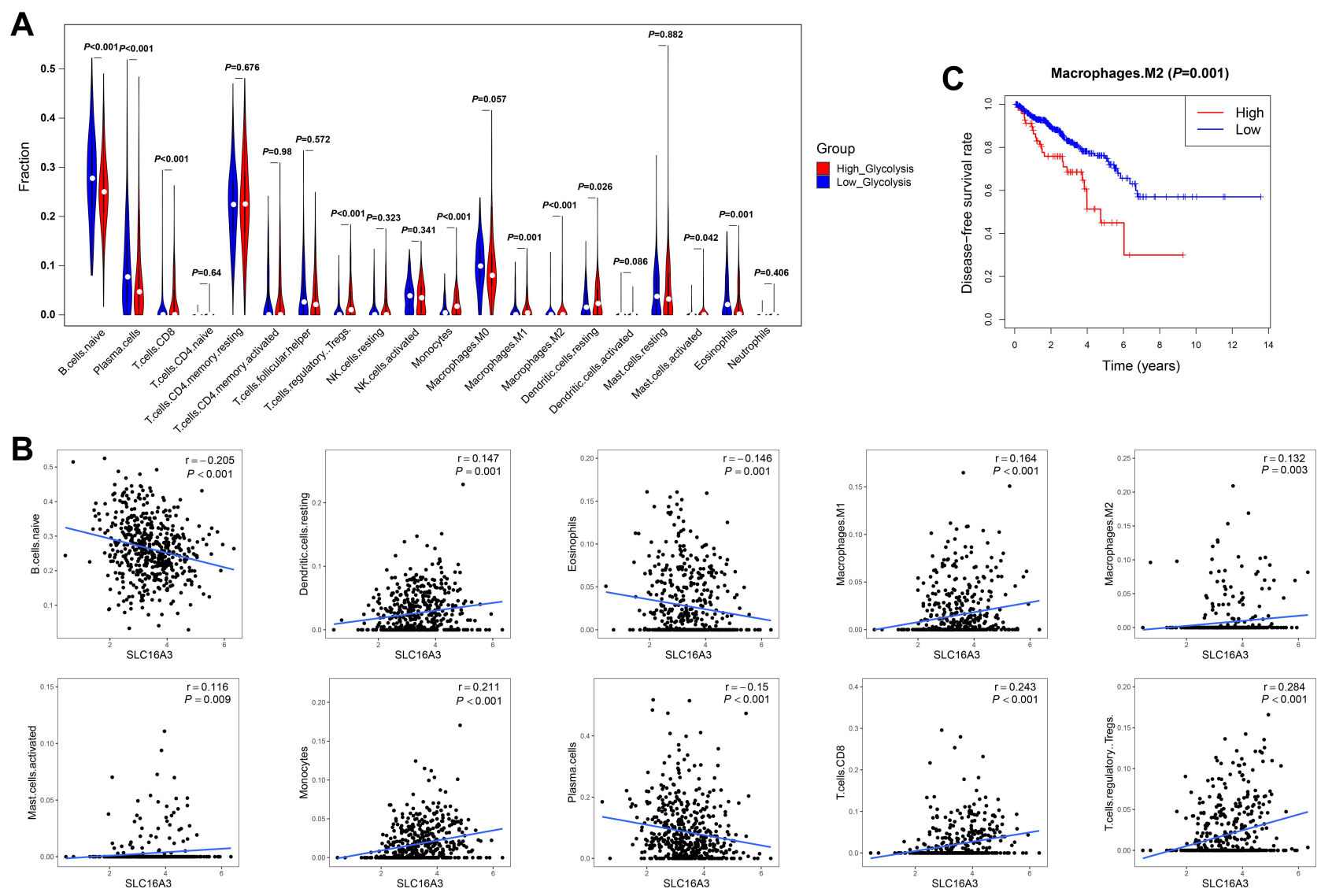

Figure 5 Relationship between immune cell infiltration and glycolytic activity. (A) Violin plot showed the degree of enrichment of tumor-infiltrating immune cell subtypes between the low- and high-glycolysis groups. (B) Spearman correlation test was used to analyze the correlation between SLCI6A3 expression and immune cell enrichment score. The Spearman correlation coefficients between SLCI6A3 expression and naïve B cells, resting dendritic cells, eosinophils, MI macrophages, M2 macrophages, activated mast cells, monocytes, plasma cells, $\mathrm{CD} 8^{+} \mathrm{T}$ cells, and regulatory $\mathrm{T}$ cells (Tregs) were remarkable. (C) Kaplan-Meier analysis showed that the group with highly enriched M2 macrophages had poorer disease-free survival rates.

resistance to anti-tumor therapy of tumor cells. ${ }^{33}$ For example, in prostate cancer, circular RNA could promote tumor progression and radioresistance by upregulating glycolysis. ${ }^{34}$ Therefore, it will be a promising treatment to reverse the metabolic state of tumor with drugs. Studies have shown that sulforaphane can prevent the occurrence of prostate cancer by inhibiting glycolysis and fatty acid metabolism. ${ }^{35,36}$ However, the search for key targets in the metabolic pathway may contribute more to precision treatment of prostate cancer. In this study, we demonstrated that SLC16A3, a key enzyme in the glycolysis pathway, was positively correlated with a higher Gleason grade and worse disease-free survival. The immunohistochemistry results also revealed that increased expression of MCT4, a plasma membrane transporter protein encoded by SLC16A3, correlated with a higher Gleason grade in prostate cancer tissues. The upregulation of glycolysis increases the levels of intracellular lactate and $\mathrm{H}^{+}$ions, thus reducing the intracellular $\mathrm{pH}$ of tumor cells. To maintain the dynamic balance of $\mathrm{pH}$ and avoid the inhibition of glycolysis by negative feedback, lactate and $\mathrm{H}^{+}$ions are excreted from the cytoplasm of tumor cells through monocarboxylate transporters, which leads to extracellular environment acidification. ${ }^{37}$ The efflux of cellular lactate/ $\mathrm{H}^{+}$ions is thought to be mediated mainly by MCT4 $4{ }^{38}$ It has been demonstrated that MCT4 is upregulated in tumors and is associated with poor prognosis in several types of cancer, ${ }^{39-42}$ including prostate cancer. ${ }^{43-46}$ MCT4 promotes the invasion of prostate cancer cells by regulating the molecules related to the VEGF/CD147/MMP9 signaling pathway. ${ }^{46}$ Knockdown of MCT4 inhibits glycolysis and tissue invasion/migration of prostate cancer cells. ${ }^{44}$ MCT4-mediated efflux disturbance usually leads to decreased levels of pyruvic and/or lactate released by glycolysis cancer cells and increased levels of intracellular pyruvate and/or lactate, resulting in cytoplasmic acidification and inhibition of glycolysis. ${ }^{32,47}$ The above 
A

10x

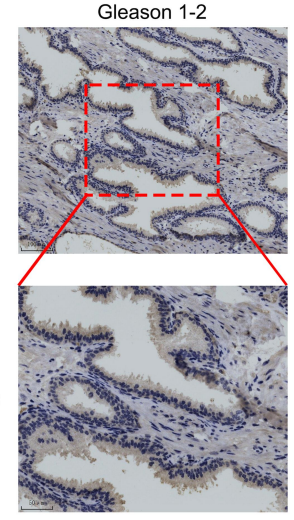

B
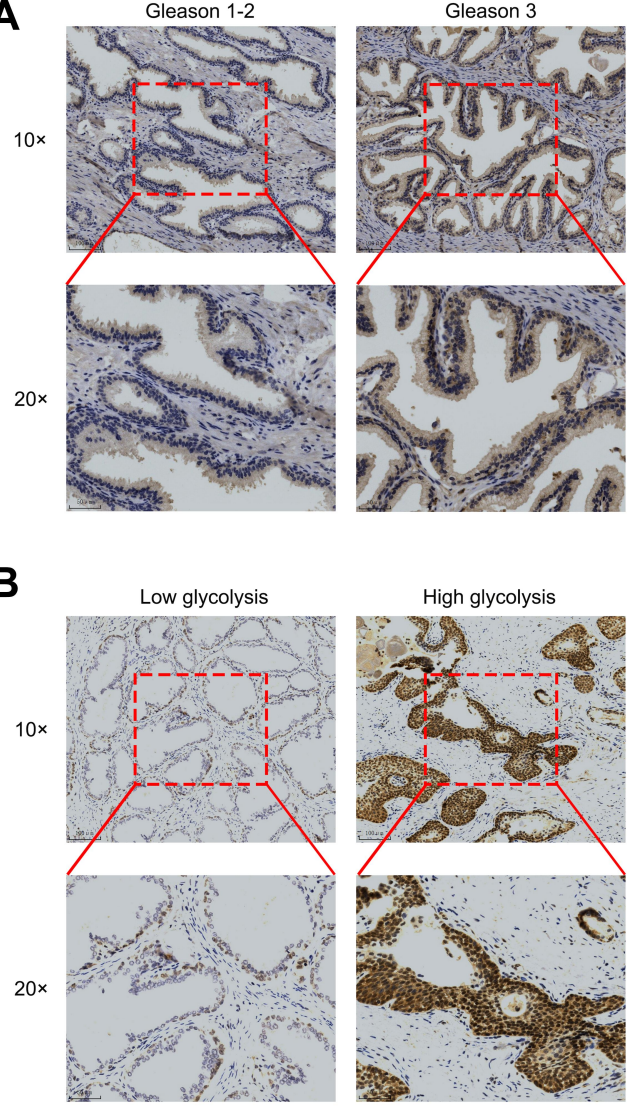

High glycolysis
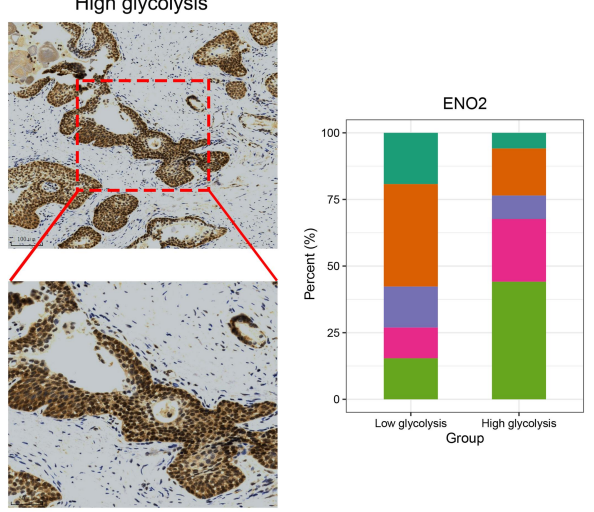

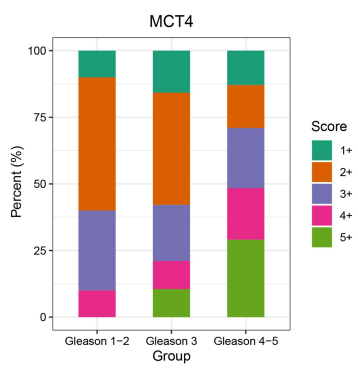

C

10x

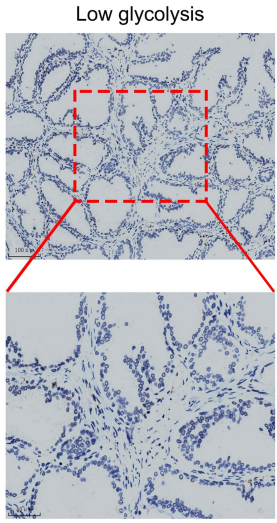

High glycolysis

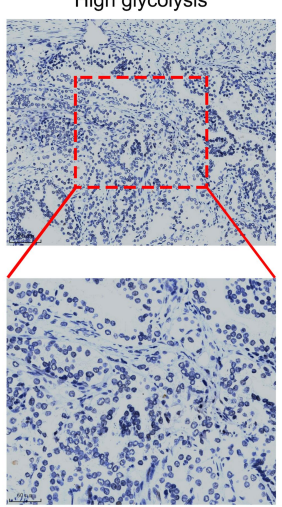

D
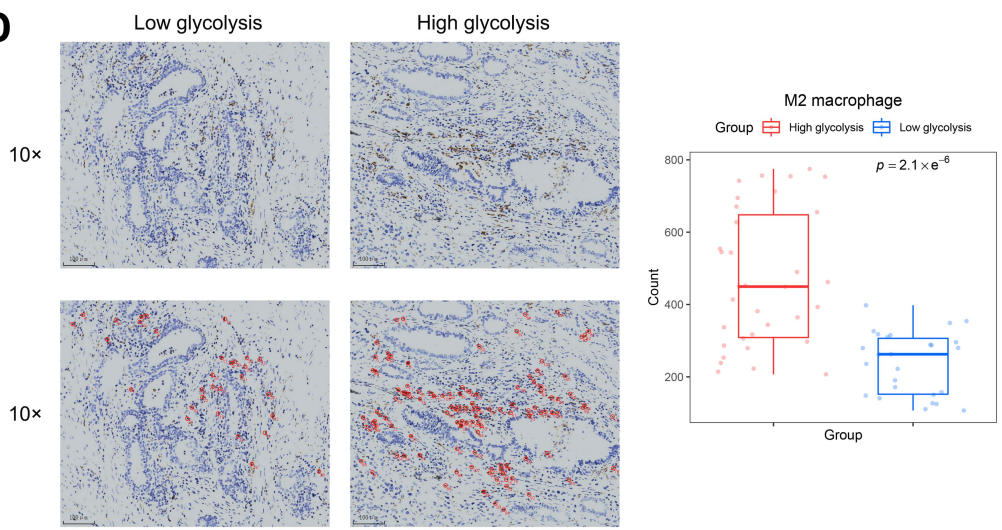

Figure 6 Immunohistochemistry results of MCT4, ENO2, PFKFB3, and CDI63. (A) Representative MCT4 immunohistochemical staining patterns in prostate cancer tissues and distribution of MCT4 immunohistochemical staining score in prostate cancer tissues according to Gleason grade. (B) Representative ENO2 immunohistochemical staining patterns in prostate cancer tissues and distribution of ENO2 immunohistochemical staining score in prostate cancer tissues according to glycolysis level. (C) Representative PFKFB3 immunohistochemical staining patterns in prostate cancer tissues. (D) Representative CDI63 immunohistochemical staining patterns in prostate cancer tissues with red circles demonstrating the selection of $\mathrm{CDI} 63^{+}$cells ( $\mathrm{M} 2$ macrophages) under low magnification. Box plots showed the number of $\mathrm{M} 2$ macrophages in the low- and high-glycolysis groups in the entire prostate cancer tissue specimen.

evidence indicates that SLC16A3 or MCT4 plays an important role in glycolysis. This study also confirmed that the expressions of some glycolysis-related genes were significantly higher in the high SLC16A3 expression group by immunohistochemistry and Western blot.
Therefore, in this study, SLC16A3 expression was considered to represent glycolytic activity.

Many studies have been conducted on tumor glycolysis, but it remains unclear how glycolysis in prostate cancer affects its immune regulation. In this study, tumor 

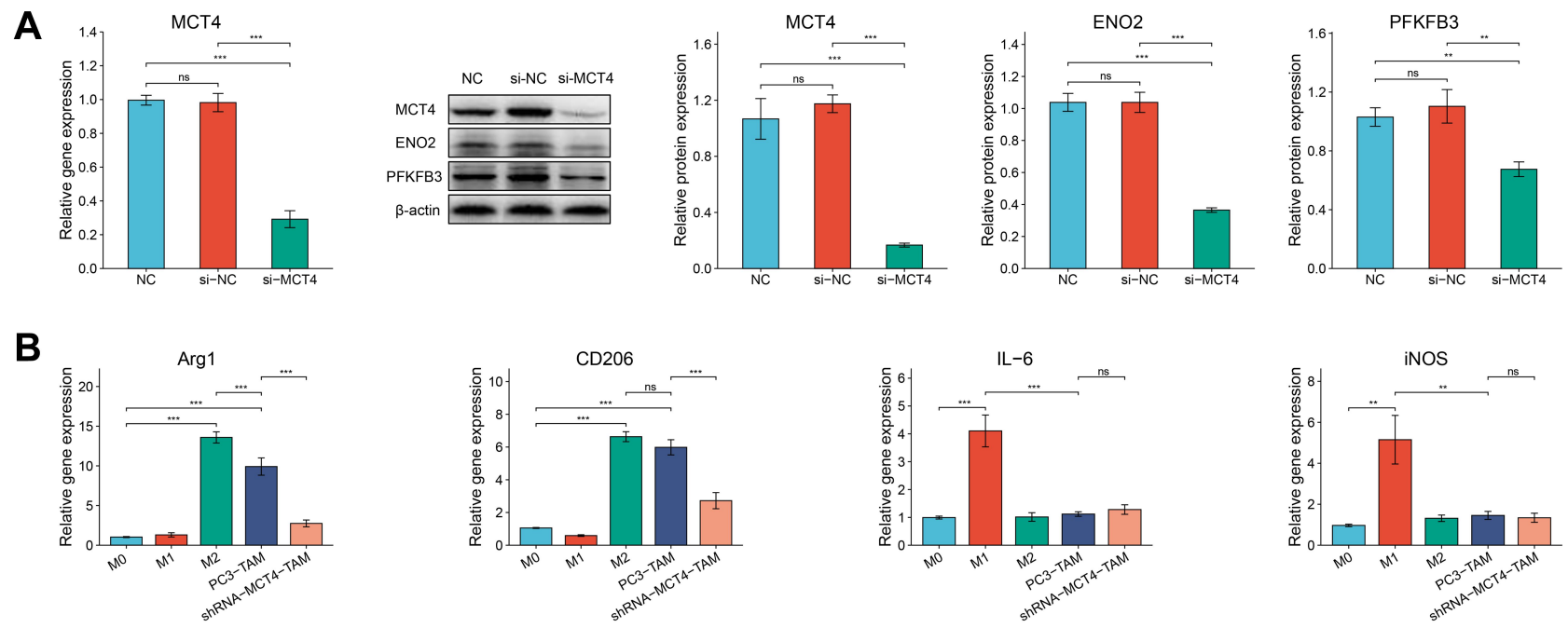

Figure 7 The relative expressions of MCT4, ENO2 and PFKFB3 in PC3 cells and the relative gene expressions of ArgI, CD206, IL-6 and iNOS in macrophages. (A) RTqPCR analysis of MCT4 gene expression of PC3 cells in NC group, si-NC group and si-MCT4 group. Western blot analysis of MCT4, ENO2 and PFKFB3 protein expressions of PC3 cells in NC group, si-NC group and si-MCT4 group. (B) Bar plots showed the gene expressions of Argl, CD206, IL-6 and iNOS measured by RT-qPCR of macrophages in M0, MI, M2, PC3-TAM and si-MCT4-PC3-TAM groups. The data are presented as the mean \pm SD values, and the error bars represent data from triplicate biological experiments. $n s, P \geq 0.05 ; * * P<0.01 ; * * * P<0.001$.

tissues were divided into high-glycolysis and lowglycolysis groups according to the expression of SLC16A3. The gene expression profiles of the two groups were compared, and functional enrichment analyses were performed. The results demonstrated that in the highglycolysis group, the expression of many cytokines and inflammatory mediators was upregulated. Further GSEA analysis revealed that the most abundant immune-related pathway in the high-glycolysis group was the IL-17 signaling pathway. IL-17 is a powerful pro-inflammatory cytokine, which has been proven to be closely related to the formation, growth, and metastasis of many types of malignant tumors. ${ }^{48-51}$ Chronic IL-17 activity results in the production of a population of pathogenic myeloid cells. $^{52}$ These myeloid cell populations play a key role in promoting the formation of pro-tumor immune microenvironment. In a series of murine cancer models, induction of IL-17-dependent pathogenic myeloid cells was associated with tumor progression, including breast cancer, ${ }^{53}$ lung cancer, ${ }^{50}$ colon cancer, ${ }^{54}$ and liver cancer. ${ }^{55}$ However, the specific mechanism by which glycolytic tumors affect the IL-17 signaling pathway remains unclear. Some studies have demonstrated that lactate from tumor cells would prompt $\mathrm{CD}^{+} \mathrm{T}$ cells to produce large amounts of IL-17, which in turn activates the IL-17 signaling pathway. ${ }^{56-58}$ Interestingly, the KEGG analysis also proved that the TNF signaling pathway was enriched in the high-glycolysis group. Previous study has found that IL-17 and TNF- $\alpha$ cooperatively promoted tumor glycolysis by regulating the expression of the glucose transporter SLC2A1 and hexokinase-2. ${ }^{59}$ IL-17 may stabilize the target gene mRNA induced by TNF- $\alpha{ }^{60}$ For the relationship between tumor glycolysis and IL-17 signaling pathways, further experimental studies are needed.

Many immune cell subgroups are essential for antitumor immunotherapy in the TME. Depending on their function and phenotype, immune cells can promote or hinder antitumor immune responses. Thus far, therapies based on immune checkpoints have not yet achieved ideal results in prostate cancer. ${ }^{61}$ Therefore, it is particularly necessary to explore immune cell infiltration in the TME of prostate cancer and its relationship with glycolysis. In this study, we compared the enrichment of immune cells between the low- and high-glycolysis groups. According to the results, the degree of enrichment of $\mathrm{CD}^{+} \mathrm{T}$ cells, Tregs, monocytes, M1 macrophages, M2 macrophages, resting DCs, and activated mast cells was significantly higher, while that of naïve $\mathrm{B}$ cells, plasma cells, and eosinophils was significantly lower in the high-glycolysis group than in the low-glycolysis group. We found that the increased infiltration of immune cells in the highglycolysis group did not lead to a good prognosis. It seemed that monocytes, macrophages, DCs, mast cells, and $\mathrm{T}$ lymphocytes established an immunosuppressive TME in prostate cancer. The existence of $\mathrm{T}$ lymphocytes in the TME is related to the prognosis and clinical 
significance of many types of cancer. Therefore, the density of $\mathrm{CD} 8^{+}$and $\mathrm{CD}^{+} \mathrm{T}$ cell infiltration at the tumor core and the invasive margin served as a basis for establishing a scoring system, known as the "Immunoscore", to evaluate the tumor immune status. ${ }^{62}$ Due to their cytotoxic function, $\mathrm{CD} 8^{+} \mathrm{T}$ cells are considered a key component of antitumor immunity. In many solid tumors, highly activated $\mathrm{CD}^{+} \mathrm{T}$ cell infiltration often indicates better prognosis. ${ }^{63,64}$ However, the correlation between $\mathrm{CD} 8^{+}$ $\mathrm{T}$ cell infiltration and prognosis is controversial in prostate cancer. A study reported that the high infiltration of $\mathrm{CD} 8^{+}$ $T$ cell predicted shorter biochemical failure-free survival in prostate cancer patients. ${ }^{65}$ Another study also found that a high proportion of $\mathrm{CD} 8^{+} \mathrm{T}$ cells was significantly associated with poor prognosis after examining the density of $\mathrm{CD} 8^{+} \mathrm{T}$ cells in 285 cases of prostate cancer. ${ }^{66}$ An analysis of gene expression data from 1567 prostate cancer samples with long-term clinical results also demonstrated that high tumor $\mathrm{CD}^{+} \mathrm{T}$ cell infiltration was associated with poor distant metastasis-free survival. ${ }^{67}$ In our study, we found that prostate cancer tissues in the high-glycolysis group with worse disease-free survival also had higher $\mathrm{CD} 8^{+}$ $\mathrm{T}$ cell infiltration, which is consistent with the results of previous studies. Evidence has shown that the TME can inhibit $\mathrm{CD}^{+} \mathrm{T}$ cell immunity, resulting in exhaustion and dysfunction of $\mathrm{CD} 8^{+} \mathrm{T}$ cells. ${ }^{68}$ Persistent antigen stimulation is the key factor leading to the exhaustion of $\mathrm{CD} 8^{+}$ $\mathrm{T}$ cells. One of the main characteristics of exhaustion is the high level of co-expression of a variety of inhibitory receptors, including PD-1, CTLA-4 and others. ${ }^{69}$ The exhaustion of $\mathrm{CD} 8^{+} \mathrm{T}$ cells finally suppresses its control of tumor. Therefore, high glycolysis of prostate cancer may indicate the exhaustion of $\mathrm{CD}^{+} \mathrm{T}$ cells infiltration, but the regulatory mechanism between them remains to be further elucidated.

In the TME, tumor cells with a highly glycolytic phenotype are likely to participate in metabolic competition with immune cells. The high level of lactate secreted by highly glycolytic tumor cells hinders the output of lactate by immune cells, thereby interfering with their metabolism and function..$^{70}$ Moreover, some immune cells may take up lactate, which impairs glycolytic function. ${ }^{71}$ On this basis, a number of studies have shown that tumor-derived lactate inhibits the proliferation, motility, and cytotoxic activity of immune cells, which ultimately inhibits immune response to tumor cells. ${ }^{56,72,73}$ Our results showed that the enrichment of M2 macrophages in the high-glycolysis group was significantly associated with poor disease-free survival in prostate cancer. Immunohistochemical experiment further confirmed that there were large amounts of M2 macrophage infiltration in prostate cancer tissue of high-glycolysis group. Moreover, we found that PC3 cells could promote M2 polarization of co-cultured macrophages and once MCT4 was knocked down, this function of PC3 cells was significantly weakened. This suggested that high glycolytic prostate cancer cells played an important role in promoting the phenotype induction of macrophages in TME. Previous studies have shown that lactate can induce phenotypic changes in macrophages, turning them into a tumor-promoting phenotype (M2-like). Lactate produced by bladder cancer cells induced M2 polarization of TAMs, while blockade of lactate flux between cancer cells and macrophages could inhibit the acquisition of this phenotype. ${ }^{74}$ Similarly, in prostate cancer, the acidic TME enhances a series of functions associated with the M2 polarization of TAMs. ${ }^{29}$ Although no further experiments were conducted to verify the effect of MCT4 expression in macrophages on M2 polarization in this article, an interesting study found that M2 macrophage polarization mainly depended on active oxidative phosphorylation rather than glycolysis. ${ }^{75}$ We hypothesize that highly glycolytic prostate cancer cells export lactate to the TME through MCT4, which in turn inhibits glycolysis of macrophages. Macrophages then acquire energy through oxidative phosphorylation, which eventually induces M2 polarization. Even though highly glycolytic tumor cells are prone to cause acidity and immunosuppression in the TME, ${ }^{10}$ further in vivo and in vitro experiments are still required to verify the mechanism of interaction between tumor glycolysis and TIICs. Our results based on public databases and bioinformatics should be interpreted with caution.

In summary, tumor glycolysis is closely related to the infiltration of immune cells in the TME. Since highly glycolytic tumors have higher immune cell infiltration, targeting tumor glycolysis may help to promote the efficacy of tumor immunotherapy by changing the function of immune cells and enhancing their antitumor immune response. $^{76}$

Furthermore, in view of the importance of MCT4 in glycolysis and tumorigenesis, MCT4 can serve as a potential therapeutic target for prostate cancer. Currently, there is no effective MCT4 inhibitor for treating malignant tumors in clinical applications. However, through the use of siRNA and antisense oligonucleotides for RNA interference experiments, it has been confirmed that inhibiting the expression of MCT4 can reduce tumor growth. ${ }^{41,44,77}$ Therefore, the development of selective 
targeted inhibitors of MCT4 may become a new therapeutic strategy for malignant tumor.

\section{Conclusion}

This study revealed that the enhancement of glycolytic activity in prostate cancer was related to increased immune cell infiltration. The IL-17 signaling pathway may play an important regulatory role in the interaction between immune cell infiltration and tumor glycolysis. Infiltration of tumorpromoting immune cells can suppress antitumor immunity in prostate cancer with high level of glycolysis. Therefore, drugs targeting glycolysis may have the effect of inhibiting tumor growth. As an important enzyme in the glycolysis pathway, MCT4 is responsible for exporting lactate from tumor cells, thereby creating an acidic TME that may influence immune cell infiltration and function. Therefore, MCT4 plays a vital role in the development of prostate cancer, which made it a potential therapeutic target. However, since most of the results in this article were based on public database through bioinformatics, the interpretation of the results requires further experiments to verify.

\section{Data Sharing Statement}

All data reported in the manuscript are from the public database, and the website can be found in Materials and methods. There are no additional unpublished data from the study.

\section{Acknowledgments}

Thanks are due to Professor Fu for assistance with the experiments.

\section{Funding}

The study was sponsored by the Science and Technology Innovation Commission Foundation of Shenzhen (Grant No. JCYJ20190808141013454 and JCYJ20180305124827261).

\section{Disclosure}

The authors report no conflicts of interest in this work.

\section{References}

1. Bray F, Ferlay J, Soerjomataram I, Siegel RL, Torre LA, Jemal A. Global cancer statistics 2018: GLOBOCAN estimates of incidence and mortality worldwide for 36 cancers in 185 countries. CA Cancer J Clin. 2018;68(6):394-424. doi:10.3322/caac.21492

2. Siegel RL, Miller KD, Jemal A. Cancer statistics, 2019. CA Cancer J Clin. 2019;69(1):7-34. doi:10.3322/caac.21551

3. Qi D, Wu C, Liu F, et al. Trends of prostate cancer incidence and mortality in Shanghai, China from 1973 to 2009. Prostate. 2015;75 (14):1662-1668. doi:10.1002/pros.23046
4. Zheng R, Sun K, Zhang S, et al. Analysis of the prevalence of malignant tumors in China in 2015. Chin J Oncol. 2019;41 (1):19-28. doi:10.3760/cma.j.issn.0253-3766.2019.01.005

5. De Angelis R, Sant M, Coleman MP, et al. Cancer survival in Europe 1999-2007 by country and age: results of EUROCARE5-a Population-Based Study. Lancet Oncol. 2014;15(1):23-34. doi:10.1016/S1470-2045(13)70546-1

6. Noone AM, Howlader N, Krapcho M, et al. SEER Cancer Statistics Review, 1975-2015. Bethesda, MD: National Cancer Institute; 2018.

7. Warburg O, Wind F, Negelein E. The metaboliam of tumors in the body. J Gen Physiol. 1927;8(6):519-530. doi:10.1085/jgp.8.6.519

8. Warburg O. On the origin of cancer cells. Science. 1956;123 (3191):309-314. doi:10.1126/science.123.3191.309

9. Pavlides S, Whitaker-Menezes D, Castello-Cros R, et al. The reverse Warburg effect: aerobic glycolysis in cancer associated fibroblasts and the tumor stroma. Cell Cycle. 2009;8(23):3984-4001. doi:10.4161/cc.8.23.10238

10. Pereira-Nunes A, Afonso J, Granja S, Baltazar F. Lactate and lactate transporters as key players in the maintenance of the Warburg effect. Adv Exp Med Biol. 2020;1219:51-74.

11. Cutruzzolà F, Giardina G, Marani M, et al. Glucose metabolism in the progression of prostate cancer. Front Physiol. 2017;8:97. doi:10.3389/fphys.2017.00097

12. Eidelman E, Twum-Ampofo J, Ansari J, Siddiqui MM. The metabolic phenotype of prostate cancer. Front Oncol. 2017;7::131. doi:10.3389/fonc.2017.00131

13. Bader DA, McGuire SE. Tumour metabolism and its unique properties in prostate adenocarcinoma. Nat Rev Urol. 2020;17(4):214-231. doi:10.1038/s41585-020-0288-x

14. Pertega-Gomes N, Felisbino S, Massie CE, et al. A glycolytic phenotype is associated with prostate cancer progression and aggressiveness: a role for monocarboxylate transporters as metabolic targets for therapy. J Pathol. 2015;236(4):517-530. doi:10.1002/path.4547

15. Yang Y. Cancer immunotherapy: harnessing the immune system to battle cancer. J Clin Invest. 2015;125(9):3335-3337. doi:10.1172/ JCI83871

16. Binnewies M, Roberts EW, Kersten K, et al. Understanding the tumor immune microenvironment (TIME) for effective therapy. Nat Med. 2018;24(5):541-550. doi:10.1038/s41591-018-0014-x

17. Nardone V, Botta $C$, Caraglia $M$, et al. Tumor infiltrating T lymphocytes expressing FoxP3, CCR7 or PD-1 predict the outcome of prostate cancer patients subjected to salvage radiotherapy after biochemical relapse. Cancer Biol Ther. 2016;17(11):1213-1220. doi:10.1080/15384047.2016.1235666

18. Erlandsson A, Carlsson J, Lundholm M, et al. M2 macrophages and regulatory $\mathrm{T}$ cells in lethal prostate cancer. Prostate. 2019;79 (4):363-369. doi:10.1002/pros.23742

19. Buck MD, Sowell RT, Kaech SM, Pearce EL. Metabolic instruction of immunity. Cell. 2017;169(4):570-586. doi:10.1016/j.cell.2017.04.004

20. Gill KS, Fernandes P, O’Donovan TR, et al. Glycolysis inhibition as a cancer treatment and its role in an anti-tumour immune response. Biochim Biophys Acta. 2016;1866(1):87-105. doi:10.1016/j. bbcan.2016.06.005

21. Gottfried E, Kunz-Schughart LA, Ebner S, et al. Tumor-derived lactic acid modulates dendritic cell activation and antigen expression. Blood. 2006;107(5):2013-2021. doi:10.1182/blood-2005-05-1795

22. Nasi A, Fekete T, Krishnamurthy A, et al. Dendritic cell reprogramming by endogenously produced lactic acid. J Immunol. 2013;191 (6):3090-3099. doi:10.4049/jimmunol.1300772

23. Pilon-Thomas S, Kodumudi KN, El-Kenawi AE, et al. Neutralization of tumor acidity improves antitumor responses to immunotherapy. Cancer Res. 2016;76(6):1381-1390. doi:10.1158/0008-5472.CAN15-1743

24. Meng J, Liu Y, Guan S, et al. The establishment of immune infiltration based novel recurrence predicting nomogram in prostate cancer. Cancer Med. 2019;8(11):5202-5213. doi:10.1002/cam4.2433 
25. Huang DW, Sherman BT, Lempicki RA. Systematic and integrative analysis of large gene lists using DAVID bioinformatics resources. Nat Protoc. 2009;4(1):44-57. doi:10.1038/nprot.2008.211

26. Liao Y, Wang J, Jaehnig EJ, Shi Z, Zhang B. WebGestalt 2019: gene set analysis toolkit with revamped UIs and APIs. Nucleic Acids Res. 2019;47(W1):W199-W205. doi:10.1093/nar/gkz401

27. Newman AM, Liu CL, Green MR, et al. Robust enumeration of cell subsets from tissue expression profiles. Nat Methods. 2015;12 (5):453-457. doi:10.1038/nmeth.3337

28. Varin A, Mukhopadhyay S, Herbein G, Gordon S. Alternative activation of macrophages by IL-4 impairs phagocytosis of pathogens but potentiates microbial-induced signalling and cytokine secretion. Blood. 2010;115(2):353-362. doi:10.1182/blood-2009-08-236711

29. El-Kenawi A, Gatenbee C, Robertson-Tessi M, et al. Acidity promotes tumour progression by altering macrophage phenotype in prostate cancer. $\mathrm{Br} J$ Cancer. 2019;121(7):556-566. doi:10.1038/ s41416-019-0542-2

30. Shen H, Sun CC, Kang L, et al. Low-dose salinomycin inhibits breast cancer metastasis by repolarizing tumor hijacked macrophages toward the M1 phenotype. Eur J Pharm Sci. 2021;157:105629. doi:10.1016/j.ejps.2020.105629

31. Zhang Y, Liu X, Liu L, et al. Upregulation of FEN1 is associated with the tumor progression and prognosis of hepatocellular carcinoma. Dis Markers. 2020;2020:2514090. doi:10.1155/2020/2514090

32. Payen VL, Mina E, Van Hée VF, Porporato PE, Sonveaux S. Monocarboxylate transporters in cancer. Mol Metab. 2020;33::48-66. doi:10.1016/j.molmet.2019.07.006

33. Pavlova NN, Thompson CB. The emerging hallmarks of cancer metabolism. Cell Metab. 2016;23(1):27-47. doi:10.1016/j. cmet.2015.12.006

34. Du S, Zhang P, Ren W, Yang F, Du C. Circ-ZNF609 accelerates the radioresistance of prostate cancer cells by promoting the glycolytic metabolism through miR-501-3p/HK2 axis. Cancer Manag Res. 2020;12:7487-7499. doi:10.2147/CMAR.S257441

35. Singh KB, Hahm ER, Alumkal JJ, et al. Reversal of the Warburg phenomenon in chemoprevention of prostate cancer by sulforaphane. Carcinogenesis. 2019;40(12):1545-1556. doi:10.1093/carcin/bgz155

36. Singh KB, Kim SH, Hahm ER, Pore SK, Jacobs BL, Singh SV. Prostate cancer chemoprevention by sulforaphane in a preclinical mouse model is associated with inhibition of fatty acid metabolism. Carcinogenesis. 2018;39(6):826-837. doi:10.1093/carcin/bgy051

37. Parks SK, Chiche J, Pouysségur J. Disrupting proton dynamics and energy metabolism for cancer therapy. Nat Rev Cancer. 2013;13 (9):611-623. doi:10.1038/nrc3579

38. Dimmer KS, Friedrich B, Lang F, Deitmer JW, Bröer S. The low-affinity monocarboxylate transporter MCT4 is adapted to the export of lactate in highly glycolytic cells. Biochem J. 2000;350(Pt 1):219-227. doi:10.1042/bj3500219

39. Chen HL, OuYang HY, Le Y, et al. Aberrant MCT4 and GLUT1 expression is correlated with early recurrence and poor prognosis of hepatocellular carcinoma after hepatectomy. Cancer Med. 2018;7 (11):5339-5350. doi:10.1002/cam4.1521

40. Fisel P, Kruck S, Winter S, et al. DNA methylation of the SLC16A3 promoter regulates expression of the human lactate transporter MCT4 in renal cancer with consequences for clinical outcome. Clin Cancer Res. 2013;19(18):5170-5181. doi:10.1158/1078-0432.CCR-13-1180

41. Todenhöfer T, Seiler R, Stewart C, et al. Selective inhibition of the lactate transporter MCT4 reduces growth of invasive bladder cancer. Mol Cancer Ther. 2018;17(12):2746-2755. doi:10.1158/1535-7163. MCT-18-0107

42. Lai SW, Lin HJ, Liu YS, Yang LY, Lu DY. Monocarboxylate transporter 4 regulates glioblastoma motility and monocyte binding ability. Cancers (Basel). 2020;12(2):380. doi:10.3390/ cancers 12020380
43. Pértega-Gomes N, Vizcaíno JR, Miranda-Gonçalves V, et al. Monocarboxylate transporter 4 (MCT4) and CD147 overexpression is associated with poor prognosis in prostate cancer. BMC Cancer. 2011;11(1):312. doi:10.1186/1471-2407-11-312

44. Choi SYC, Xue H, Wu R, et al. The MCT4 gene: a novel, potential target for therapy of advanced prostate cancer. Clin Cancer Res. 2016;22(11):2721-2733. doi:10.1158/1078-0432.CCR-15-1624

45. Choi SYC, Ettinger SL, Lin D, et al. Targeting MCT4 to reduce lactic acid secretion and glycolysis for treatment of neuroendocrine prostate cancer. Cancer Med. 2018;7(7):3385-3392. doi:10.1002/ cam4.1587

46. Sun Q, Hu LL, Fu Q. MCT4 promotes cell proliferation and invasion of castration-resistant prostate cancer PC-3 cell line. EXCLI J. 2019;18:187-194. doi:10.17179/excli2018-1879

47. Dhup S, Dadhich RK, Porporato PE, Sonveaux P. Multiple biological activities of lactic acid in cancer: influences on tumor growth, angiogenesis and metastasis. Curr Pharm Des. 2012;18(10):1319-1330. doi:10.2174/138161212799504902

48. Wang K, Kim MK, Di Caro G, et al. Interleukin-17 receptor a signaling in transformed enterocytes promotes early colorectal tumorigenesis. Immunity. 2014;41(6):1052-1063. doi:10.1016/j. immuni.2014.11.009

49. Zhang Y, Zoltan M, Riquelme E, et al. Immune cell production of interleukin 17 induces stem cell features of pancreatic intraepithelial neoplasia cells. Gastroenterology. 2018;155(1):210-223.e213. doi:10.1053/j.gastro.2018.03.041

50. Jin C, Lagoudas GK, Zhao C, et al. Commensal microbiota promote lung cancer development via $\gamma \delta$ T cells. Cell. 2019;176(5):998-1013. e1016. doi:10.1016/j.cell.2018.12.040

51. Chen X, Cai G, Liu C, et al. IL-17R-EGFR axis links wound healing to tumorigenesis in Lrig1 $^{+}$stem cells. J Exp Med. 2019;216 (1):195-214. doi:10.1084/jem.20171849

52. Veglia F, Perego M, Gabrilovich D. Myeloid-derived suppressor cells coming of age. Nat Immunol. 2018;19(2):108-119. doi:10.1038/ s41590-017-0022-X

53. Coffelt SB, Kersten K, Doornebal CW, et al. IL-17-producing $\gamma \delta$ $\mathrm{T}$ cells and neutrophils conspire to promote breast cancer metastasis. Nature. 2015;522(7556):345-348. doi:10.1038/nature14282

54. Thiele Orberg E, Fan H, Tam AJ, et al. The myeloid immune signature of enterotoxigenic Bacteroides fragilis-induced murine colon tumorigenesis. Mucosal Immunol. 2017;10(2):421-433. doi:10.1038/mi.2016.53

55. Ma S, Cheng Q, Cai Y, et al. IL-17A produced by $\gamma \delta$ T cells promotes tumor growth in hepatocellular carcinoma. Cancer Res. 2014;74 (7):1969-1982. doi:10.1158/0008-5472.CAN-13-2534

56. Haas R, Smith J, Rocher-Ros V, et al. Lactate regulates metabolic and pro-inflammatory circuits in control of $\mathrm{T}$ cell migration and effector functions. PLoS Biol. 2015;13(7):e1002202. doi:10.1371/journal. pbio. 1002202

57. Shime H, Yabu M, Akazawa T, et al. Tumor-secreted lactic acid promotes IL-23/IL-17 proinflammatory pathway. J Immunol. 2008;180(11):7175-7183. doi:10.4049/jimmunol.180.11.7175

58. Yabu M, Shime H, Hara H, et al. IL-23-dependent and independent enhancement pathways of IL-17A production by lactic acid. Int Immunol. 2011;23(1):29-41. doi:10.1093/intimm/ dxq455

59. Straus DS. TNF $\alpha$ and IL-17 cooperatively stimulate glucose metabolism and growth factor production in human colorectal cancer cells. Mol Cancer. 2013;12:78. doi:10.1186/1476-4598-12-78

60. Hamilton T, Li X, Novotny M, et al. Cell type- and stimulus-specific mechanisms for post-transcriptional control of neutrophil chemokine gene expression. J Leukoc Biol. 2012;91(3):377-383. doi:10.1189/ jlb.0811404 
61. Jansen CS, Prokhnevska N, Kissick HT. The requirement for immune infiltration and organization in the tumor microenvironment for successful immunotherapy in prostate cancer. Urol Oncol. 2019;37 (8):543-555. doi:10.1016/j.urolonc.2018.10.011

62. Mlecnik B, Bindea G, Angell HK, et al. Integrative analyses of colorectal cancer show Immunoscore is a stronger predictor of patient survival than microsatellite instability. Immunity. 2016;44 (3):698-711. doi:10.1016/j.immuni.2016.02.025

63. Sato E, Olson SH, Ahn J, et al. Intraepithelial CD8+ tumor-infiltrating lymphocytes and a high $\mathrm{CD} 8+/$ regulatory $\mathrm{T}$ cell ratio are associated with favorable prognosis in ovarian cancer. Proc Natl Acad Sci USA. 2005;102(51):18538-18543. doi:10.1073/ pnas.0509182102

64. Mahmoud SMA, Paish EC, Powe DG, et al. Tumor-infiltrating CD8+ lymphocytes predict clinical outcome in breast cancer. J Clin Oncol. 2011;29(15):1949-1955. doi:10.1200/JCO.2010.30.5037

65. Ness N, Andersen S, Valkov A, et al. Infiltration of CD8+ lymphocytes is an independent prognostic factor of biochemical failure-free survival in prostate cancer. Prostate. 2014;74(14):1452-1461. doi:10.1002/pros.22862

66. Leclerc BG, Charlebois R, Chouinard G, et al. CD73 expression is an independent prognostic factor in prostate cancer. Clin Cancer Res. 2016;22(1):158-166. doi:10.1158/1078-0432.CCR-15-1181

67. Zhao SG, Lehrer J, Chang SL, et al. The immune landscape of prostate cancer and nomination of PD-L2 as a potential therapeutic target. J Natl Cancer Inst. 2019;111(3):301-310. doi:10.1093/jnci/ djy 141

68. Apetoh L, Smyth MJ, Drake CG, et al. Consensus nomenclature for $\mathrm{CD}^{+} \mathrm{T}$ cell phenotypes in cancer. Oncoimmunology. 2015;4(4): e998538. doi:10.1080/2162402X.2014.998538

69. Kurachi M. CD8(+) T cell exhaustion. Semin Immunopathol. 2019;41 (3):327-337. doi:10.1007/s00281-019-00744-5
70. Romero-Garcia S, Moreno-Altamirano MMB, Prado-Garcia H, Sánchez-García FJ. Lactate contribution to the tumor microenvironment: mechanisms, effects on immune cells and therapeutic relevance. Front Immunol. 2016;7:52. doi:10.3389/ fimmu.2016.00052

71. Hargadon KM. Strategies to improve the efficacy of dendritic cell-based immunotherapy for melanoma. Front Immunol. 2017;8:1594. doi:10.3389/fimmu.2017.01594

72. Husain Z, Huang Y, Seth P, Sukhatme VP. Tumor-derived lactate modifies antitumor immune response: effect on myeloid-derived suppressor cells and NK cells. J Immunol. 2013;191(3):1486-1495. doi:10.4049/jimmunol.1202702

73. Husain Z, Seth P, Sukhatme VP. Tumor-derived lactate and myeloid-derived suppressor cells: linking metabolism to cancer immunology. Oncoimmunology. 2013;2(11):e26383. doi:10.4161/ onci.26383

74. Zhao Y, Wang D, Xu T, et al. Bladder cancer cells re-educate TAMs through lactate shuttling in the microfluidic cancer microenvironment. Oncotarget. 2015;6(36):39196-39210. doi:10.18632/oncotarget.5538

75. Wang F, Zhang S, Vuckovic I, et al. Glycolytic stimulation is not a requirement for M2 macrophage differentiation. Cell Metab. 2018;28(3):463-475.e464. doi:10.1016/j.cmet.2018.08.012

76. Bénéteau M, Zunino B, Jacquin MA, et al. Combination of glycolysis inhibition with chemotherapy results in an antitumor immune response. Proc Natl Acad Sci USA. 2012;109(49):20071-20076. doi:10.1073/pnas.1206360109

77. Kim HK, Lee I, Bang H, et al. MCT4 expression is a potential therapeutic target in colorectal cancer with peritoneal carcinomatosis. Mol Cancer Ther. 2018;17(4):838-848. doi:10.1158/1535-7163.MCT-17-0535

\section{Publish your work in this journal}

OncoTargets and Therapy is an international, peer-reviewed, open access journal focusing on the pathological basis of all cancers, potential targets for therapy and treatment protocols employed to improve the management of cancer patients. The journal also focuses on the impact of management programs and new therapeutic agents and protocols on patient perspectives such as quality of life, adherence and satisfaction. The manuscript management system is completely online and includes a very quick and fair peer-review system, which is all easy to use. Visit http://www.dovepress.com/ testimonials.php to read real quotes from published authors. 